\title{
The Optical Excitonic Aharonov-Bohm Effect in a Few Nanometer Wide Type-I Nanorings*
}

\author{
M. TAdiĆ $\bar{c}^{a \dagger}$, V. Arsoski ${ }^{a}$, N. $\check{C ̆ U k A R I C ́}^{a}$ And F.M. Peeters ${ }^{b}$ \\ ${ }^{a}$ Faculty of Electrical Engineering, University of Belgrade, P.O. Box 3554, 11120 Belgrade, Serbia \\ ${ }^{b}$ Department of Physics, University of Antwerp, Groenenborgerlaan 171, B-2020 Antwerp, Belgium
}

(Received October 27, 2010)

\begin{abstract}
The optical excitonic Aharonov-Bohm effect in type-I three-dimensional (In,Ga)As/GaAs nanorings is theoretically explored. The single-particle states of the electron and the hole are extracted from the effective mass theory in the presence of inhomogeneous strain, and an exact numerical diagonalization approach is used to compute the exciton states and the oscillator strength $f_{x}$ for exciton recombination. We studied both the large lithographically-defined and small self-assembled rings. Only in smaller self-assembled nanorings we found optical excitonic Aharonov-Bohm effect. Those oscillations are established by anticrossings between the optically active exciton states with zero orbital momentum. In lithographically defined rings, whose average radius is $33 \mathrm{~nm}, f_{x}$ shows no oscillations, whereas in the smaller self-assembled nanoring with average radius of $11.5 \mathrm{~nm}$ oscillations in $f_{x}$ for the ground exciton state are found as function of the magnetic field that is superposed on a linear dependence. These oscillations are smeared out at finite temperature, thus photoluminescence intensity exhibits step-like variation with magnetic field even at temperature as small as $4.2 \mathrm{~K}$.
\end{abstract}

PACS numbers: 73.21.La, 78.67.Hc

\section{Introduction}

Recently, it has been demonstrated that certain modifications of the Stranski-Krastanov growth sequence can change the morphology of lens-shaped quantum dots into quantum rings [1-3]. An external magnetic field induces angular momentum transitions in the electron ground state of such quantum rings. Excitonic Aharonov-Bohm $(A B)$ effect was predicted to occur in one-dimensional (1D) rings [4]. Because of angular momentum transitions and the dependence of the excitonic absorption on the orbital symmetry, a bright exciton ground state may revert to dark when the magnetic field increases, which is called the optical excitonic Aharonov-Bohm effect [5]. This effect was predicted for the electron and hole composing the polarized exciton, where the two particles are located on concentric one-dimensional rings, and for the case of weak binding, i.e. when the kinetic energies of the particles exceed the Coulomb interaction potential. The exact treatments and numerical calculations indicated that the exciton energy $\mathrm{AB}$ oscillations are absent in $2 \mathrm{D}$ rings, while there is no clear experimental verifica-

\footnotetext{
* Presented at the International School and Conference on Photonics PHOTONICA09, Belgrade, Serbia, August 24-28, 2009

$\dagger$ corresponding author; e-mail: tadic@etf.bg.ac.rs
}

tion of the optical excitonic $\mathrm{AB}$ effect in 3D nanorings. In addition to self-assembled (In,Ga)As rings (SAR's) [1] a beautiful experiment of photoluminescence characterization of lithographically-defined rings (LDR's) in magnetic field was recently realized in Ref. [6]. These rings are larger than the fabricated SAR's.

In the present study, we employ the single-band effective-mass model to compute the single-particle states in a strained (In,Ga)As nanoring of rectangular vertical profile. Inhomogeneous strain is present in the structure, which as recently found facilitates growth of rings in vertical stacks $[7,8]$ similar to the case of smaller quantum dots. Strain changes the symmetry of the crystal and causes splitting of the zone center states in the valence band. The later effect justifies the use of the single-band approach to compute the hole states. Because the energy of the heavy hole states increases due to strain, the lowest energy exciton states are mainly heavy-hole like. The effective-mass Hamiltonian offers the single-particle states as a convenient basis for the computation of the exciton states. The magnetic field is varied to explore the $\mathrm{AB}$ effect and to investigate the effect on the oscillator strength for exciton recombination. Our aim is to investigate whether excitonic and optical excitonic $\mathrm{AB}$ effects are present in $3 \mathrm{D}$ nanorings, and also to find $\mathrm{AB}$ oscillations in the oscillator strength.

The paper is organized as follows. In Sect. 2 the theoretical models of strain, the electronic states, and the 
optical transitions are presented. The numerical results for the LDR and SAR are given and the magnetooptical properties of the two are discussed and compared in Sect. 3 .

\section{Theoretical model}

The analyzed rings have rectangular vertical profile, with inner radius $R_{1}$, outer radius $R_{2}$, and height $h$. The strain distribution in the ring is extracted from the continuum mechanical model [8]. The electron and hole states are modeled by the single-band effective-mass theory [8], while the exact diagonalization approach extracts the exciton states from the secular equation

$$
\begin{aligned}
& \left(E_{\mathrm{e} k}-E_{\mathrm{h} m}-E_{x}\right) \delta_{i k} \delta_{j m} \\
& \quad+\sum_{k} \sum_{m}\left\langle\psi_{\mathrm{e} i} \psi_{\mathrm{h} j}\left|V_{\mathrm{C}}\right| \psi_{\mathrm{e} k} \psi_{\mathrm{h} m}\right\rangle=0,
\end{aligned}
$$

where $V_{\mathrm{C}}=-e^{2} / 4 \pi \varepsilon_{s} \varepsilon_{0}\left(\boldsymbol{r}_{\mathrm{e}}-\boldsymbol{r}_{\mathrm{h}}\right)$ denotes the Coulomb potential energy between the electron and the hole, $\psi_{\mathrm{e} k}$ and $\psi_{\mathrm{h} m}$ are the electron and hole wave functions, respectively, and $E_{x}$ is the exciton energy. Here, the state is labeled by $i=\left(n_{\mathrm{e}}, l_{\mathrm{e} i}\right)$. A straightforward derivation gives

$$
\begin{aligned}
& \left\langle\psi_{\mathrm{e} i} \psi_{\mathrm{h} j}\left|V_{\mathrm{C}}\right| \psi_{\mathrm{e} k} \psi_{\mathrm{h} m}\right\rangle=\frac{\mathrm{e}^{2}}{4 \pi^{2} \varepsilon_{s}} \delta_{l_{\mathrm{e} i}+l_{\mathrm{h} j}=l_{\mathrm{e} k}+l_{\mathrm{h} m}} \\
& \times \int_{0}^{\infty} k_{\|} \mathrm{d} k_{\|} \int_{-\infty}^{+\infty} \mathrm{d} k_{z} \frac{1}{k_{\|}^{2}+k_{z}^{2}} \\
& +\Phi_{\mathrm{e}}\left(k_{\|},-k_{z}\right) \Phi_{\mathrm{h}}\left(k_{\|}, k_{z}\right)
\end{aligned}
$$

where $k_{\|}$and $k_{z}$ denote the in-plane and the $z$ component of the wave vector in the Fourier space, respectively. $\Phi_{\mathrm{e}}$ is the two-dimensional transform given by

$$
\begin{aligned}
& \Phi_{\mathrm{e}}\left(k_{\|}, k_{z}\right) \\
& =\int_{0}^{\infty} \rho \mathrm{d} \rho \int_{-\infty}^{+\infty} \mathrm{d} z \psi_{\mathrm{e} i}^{*} \psi_{\mathrm{e} k} J_{\left|l_{i}-l_{k}\right|}\left(k_{\|} \rho\right) \mathrm{e}^{\mathrm{i} k_{z} z} .
\end{aligned}
$$

$L=l_{\mathrm{e}}+l_{\mathrm{h}}$ is a good quantum number for the exciton as indicated by the Kronecker $\delta$ in Eq. (2). The other good quantum numbers of the exciton states are the principal quantum number $n$ and the parity of the exciton $\sigma_{x}$. Therefore, the exciton states are labeled by $n L^{\sigma_{x}}$.

From the exciton wave functions we obtain the value of the oscillator strength for excitonic absorption,

$$
f_{x}=\sum_{k} \sum_{m} c_{k m} f_{k m} .
$$

Here $c_{k m}$ denotes the expansion coefficient of the product function of the electron and hole wave functions $\psi_{k} \psi_{m}$, and $f_{k m}$ is the oscillator strength between the single-particle electron and hole states,

$$
f_{k m}=\frac{2}{m_{0} E_{k m}}\left|M_{k m}\right|^{2},
$$

where $m_{0}$ denotes the free-electron mass, $E_{k m}=E_{k}-E_{m}$ is the transition energy, and $M_{k m}$ is the momentum transition matrix element

$$
M_{k m}=\left\langle S \sigma_{\mathrm{e}}|\varepsilon \cdot \boldsymbol{p}| u_{\mathrm{hh}} \sigma_{\mathrm{hh}}\right\rangle m_{k m},
$$

where $m_{k m}=\left\langle\psi_{\mathrm{e} k} \mid \psi_{\mathrm{h} m}\right\rangle$ is determined as the overlap integral between the two envelope functions, and $\left\langle S \sigma_{\mathrm{e}}|\varepsilon \cdot \boldsymbol{p}| u_{\mathrm{hh}} \sigma_{\mathrm{hh}}\right\rangle$ is the matrix element between the zone-center electron and hole states $S$ and $u_{\mathrm{hh}}$, respectively. The spins of the electron and the heavy hole are denoted by $\sigma_{\mathrm{e}}$ and $\sigma_{\mathrm{hh}}$, and because of selection rules, transitions are allowed only between the zone-center states of equal spins and for the polarization vector of incoming light $\varepsilon$ oriented in the $x y$ plane. Furthermore, only states of equal parity and opposite orbital momentum produce finite single particle transition matrix elements. Hence, only $L=0$ states are bright exciton states. Furthermore, the exciton states are statistically distributed, and an appropriate figure of merit for experiments at finite temperature is the average oscillator strength

$$
\left\langle f_{x}\right\rangle=\frac{\sum_{i} f_{x i} \mathrm{e}^{-E_{x i} / k_{\mathrm{B}} T}}{\sum_{i} \mathrm{e}^{-E_{x i} / k_{\mathrm{B}} T}},
$$

which will be referred to as photoluminescence intensity [9].

\section{Numerical results and discussion}

We consider the experimental nanorings analyzed in Refs. [6] and [10] and took $T=4.2 \mathrm{~K}$. The LDR has inner radius of $R_{1}=19 \mathrm{~nm}$, outer radius of $R_{2}=46 \mathrm{~nm}$, height $h=7 \mathrm{~nm}$, and only $10 \%$ of InAs is found to be present in the alloy inside the ring [6]. The SAR, which was fabricated and analyzed in Ref. [10], has inner radius of $8 \mathrm{~nm}$, outer radius of $15 \mathrm{~nm}$, height $h=4 \mathrm{~nm}$ and contains $55 \%$ of InAs.

Figure 1 displays the lowest energy $L=0$ and $L=-1$ energy levels in the LDR, shown by the solid and dashed lines, as function of the magnetic field. The lowest exciton energies for both displayed $L$ 's are separated from the higher states by about $6 \mathrm{meV}$. The Aharonov-Bohm oscillations are obviously not present, or are very weak, in the ground exciton state. The observed diamagnetic shift in the range from 0 to $6 \mathrm{~T}$ is about $2 \mathrm{meV}$, which agree well with the experimental finding of Ref. [6]. We note here that the experiment of Ref. [6] was not able to find any $\mathrm{AB}$ oscillations, which is also a result of our calculation. The higher exciton states anticross, which leads to oscillations in these energy levels as they depend on $B$. These anticrossings correspond to flux quantization. As an example, the second and the third exciton states anticross with the period of $1.4 \mathrm{~T}$. These anticrossings correspond to integer number of flux quanta threading the ring (see Ref. [11]). The radius of the equivalent $1 \mathrm{D}$ ring extracted from the locations of these crossings amounts to $31 \mathrm{~nm}$. It indicates that the exciton stays close to the center of the ring.

Dependence of the oscillator strength for recombination of the ground exciton state in the LDR is shown in Fig. 2a. Decrease of the oscillator strength for $B>2 \mathrm{~T}$ is due to less localized electron and hole states in the higher single-particle states. Notice that both the electron and hole states show orbital momentum transitions 


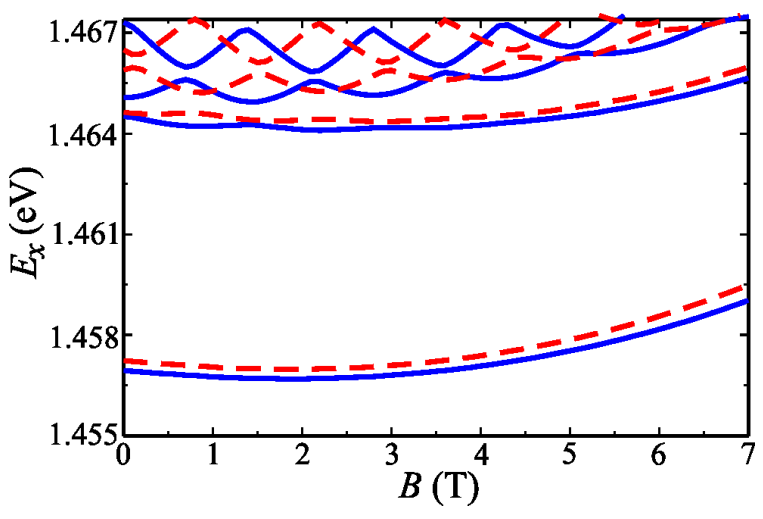

Fig. 1. Variation of the $L=0$ (solid lines) and $L=-1$ (dashed lines) even exciton energy levels in the LDR with magnetic field. Aharonov-Bohm oscillations are not present (or are negligible) in the ground state.

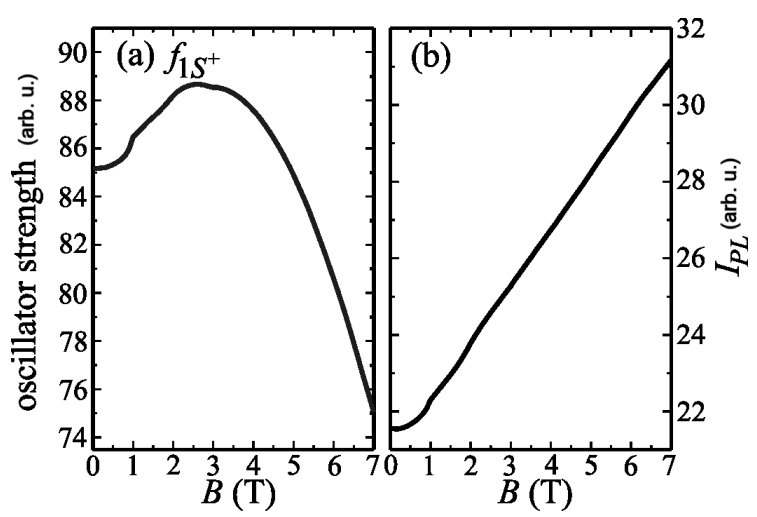

Fig. 2. (a) The oscillator strength for recombination of the $1 S^{+}$exciton states in the LDR as a function of magnetic field. (b) The photoluminescence intensity as a function of $B$.

with increasing magnetic field. The composition changes at about $1 \mathrm{~T}$, which is slightly larger than the location of the first orbital momentum transition. Below $1 \mathrm{~T}$, the maximum contribution to the ground exciton state arises from the $s$ states of the electron and the hole. In the range from 1 to $3 \mathrm{~T}$, the maximum contribution is due to the electron $l=-1$ and the hole $l=+1$ states. These single particle states are less localized, which leads to a decrease of the oscillator strength. Furthermore, the variation is not smooth close to the change of composition of the exciton states, i.e. at 1 and $3 \mathrm{~T}$. On the other hand, the photoluminescence intensity shown in Fig. 2b, increases quasi-linearly in the whole range of $B$. The main contribution to $I_{\mathrm{PL}}$ arises from the lowest energy bright exciton states, i.e. those having equal spins of the electron and hole. The separation between those states and other which are not bright increases when magnetic field increases, which leads to a decrease of the denominator of Eq. (7). Similar increase was previously found in $2 \mathrm{D}$ rings [11].

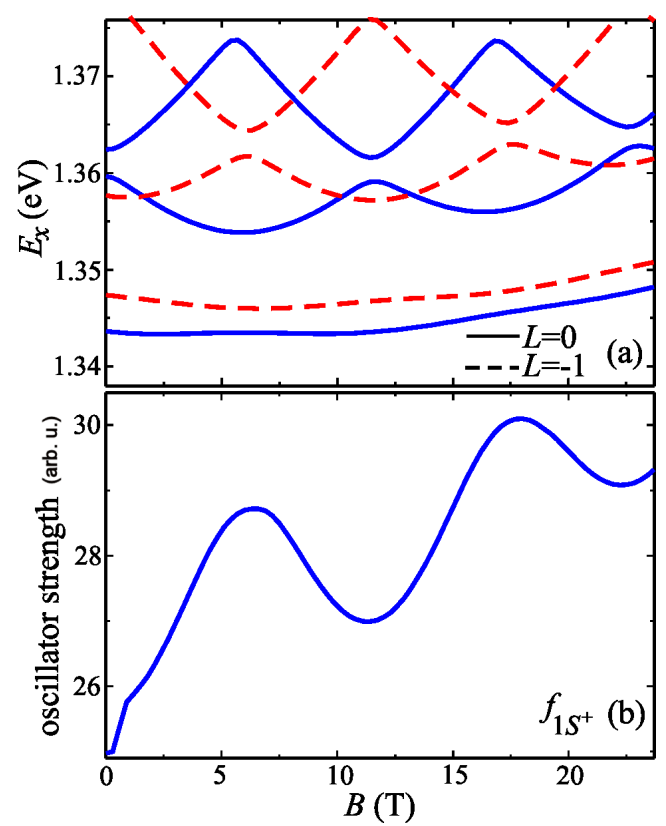

Fig. 3. (a) The $L=0$ (solid lines) and $L=-1$ (dashed lines) exciton energy levels in the SAR as function of $B$. (b) The oscillator strength for recombination of the ground exciton state exhibits oscillations.

When the average radius of the ring decreases, both the period and amplitude of the Aharonov-Bohm oscillations in the higher exciton states increase (see Fig. 3a) The period of these oscillations in the SAR is $11.5 \mathrm{~T}$, which corresponds to a radius of about $10.7 \mathrm{~nm}$, and as similar to the larger LDR is very close to the average radius of the ring. However, the appreciable oscillations arise also in the ground exciton state. Therefore, when the lateral dimensions of type-I rings decrease, the amplitude of the oscillations in the ground state increases.

In addition to the energy of the ground exciton state, the oscillator strength for its recombination in the SAR oscillates with $B$, as shown in Fig. 3b. This implies that the optical excitonic Aharonov-Bohm effect arises (or is enhanced) in type-I rings when their lateral dimensions decrease. The minima of the oscillator strength occur at 11.5 and $23 \mathrm{~T}$, which correspond to positions of anticrossings between the exciton states. When two states anticross, the exciton wave functions interchange their spatial localization, which leads to a local minimum of the oscillator strength.

In order to explore the observed excitonic AharonovBohm oscillations in Fig. 3a, we show in Fig. 4a detail of the variation of the ground exciton state energy variation with magnetic field around its first anticrossing. The ground exciton energy level varies by $0.15 \mathrm{meV}$ around the anticrossing at $6 \mathrm{~T}$.

Although the oscillations of the ground exciton energy level are present in the dependence of the oscillator strength for the recombination of the ground exciton energy level, as shown in Fig. 3b, the photoluminescence 


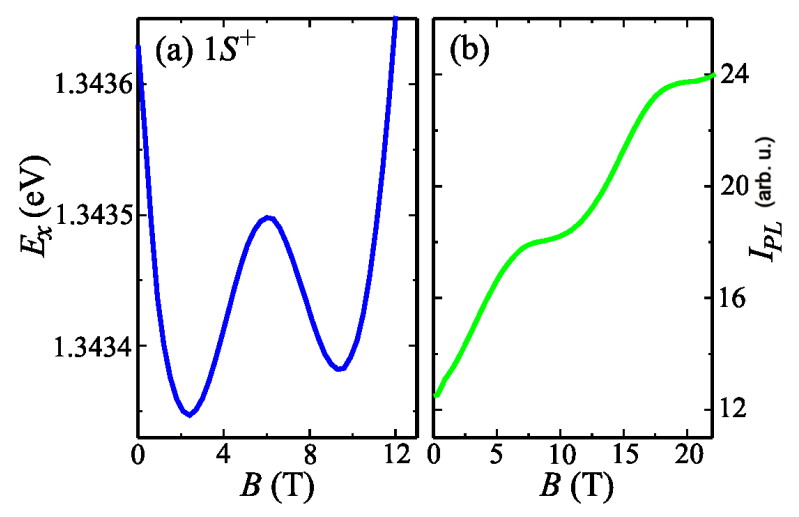

Fig. 4. (a) Detail of the dependence of the ground exciton energy in the SAR on magnetic field, around the position of the first anticrossing. The energy level variation is about $0.15 \mathrm{meV}$. (b) The photoluminescence intensity shows a step like variation as function of $B$.

intensity in the SAR does not oscillate, as shown in Fig. 4b. Rather, it exhibits step-like features. These steps were not present in the dependence of $I_{\mathrm{PL}}$ in the larger LDR shown in Fig. 2b. We might infer that the reduction of temperature below $4.2 \mathrm{~K}$, and possibly the average diameter of the ring could lead to oscillations in the dependence of $I_{\mathrm{PL}}$ on $B$. The effect of decreasing temperature would be to decrease the population of the higher exciton states, while the decreasing lateral dimensions could put the exciton in the regime of even stronger confinement. The optical excitonic Aharonov-Bohm effect is indeed predicted to occur for the case of strong confinement in concentric $1 \mathrm{D}$ rings [5].

\section{Conclusion}

We present a model of the exciton states and the oscillator strength for recombination of an exciton in strained three-dimensional nanorings in the presence of a perpendicular magnetic field. Two experimental structures, considered previously in Refs. [6] and [10], were studied. No oscillations of the ground exciton energy level in large LDR [6] are observed (or are extremely small), while in the smaller SAR's [10] the ground exciton energy level exhibits oscillations as function of $B$. The photoluminescence intensity in the larger ring increases quasi-linearly with magnetic field, while step-like features are produced in the smaller SAR at a temperature of $4.2 \mathrm{~K}$. These steps arise from the optical Aharonov-Bohm effect in the ground exciton state. The oscillations in this energy level are due to anticrossings with the second $L=0$ states. However, their amplitude is of the order of $0.15 \mathrm{meV}$. The observed variations in the electronic structure lead to local minima of the oscillator strength for recombination of the ground exciton state with $B$. We conclude that a decrease of the lateral dimensions of type-I rings could lead to (or enhance) oscillations which mark the optical excitonic Aharonov-Bohm effect in the ground exciton state.

\section{References}

[1] J.M. Garcia, G. Medeiros-Ribeiro, K. Schmidt, T. Ngo, J.L. Feng, A. Lorke, J. Kotthaus, P.M. Petroff, Appl. Phys. Lett. 71, 2014 (1997).

[2] B.C. Lee, O. Voskoboynikov, C.P. Lee, Physica E 24, 87 (2004).

[3] A. Lorke, R. Johannes Luyken, A.O. Govorov, J.P. Kotthaus, J.M. Garcia, P.M. Petroff, Phys. Rev. Lett. 84, 2223 (2000).

[4] R.A. Römer, M.E. Raikh, Phys. Rev. B 62, 7045 (2000).

[5] A.O. Govorov, S.E. Ulloa, K. Karrai, R.J. Warburton, Phys. Rev. B 66, 081309(R) (2002).

[6] M. Bayer, M. Korkusinski, P. Hawrylak, T. Gutbrod, M. Michel, A. Forchel, Phys. Rev. Lett. 90, 186801 (2003).

[7] D. Granados, J.M. Garcia, T. Ben, S.I. Molina, Appl. Phys. Lett. 86, 071918 (2005).

[8] M. Tadić, F.M. Peeters, Phys. Rev. B 79, 153305 (2009).

[9] M.H. Degani, M.Z. Maialle, G. Medeiros-Ribeiro, E. Ribeiro, Phys. Rev. B 78, 075322 (2008).

[10] N.A.J.M. Kleemans, I.M.A. Bominaar-Silkens, V.M. Fomin, V.N. Gladilin, D. Granados, A.G. Taboada, J.M. Garcia, P. Offermans, U. Zeitler, P.C.M. Christianen, C.J. Maan, J.T. Devreese, P.M. Koenraad, Phys. Rev. Lett. 99, 146808 (2007).

[11] H. Hu, J.-L. Zhu, D.-J. Li, J.-J. Xiong, Phys. Rev. B 63, 195307 (2001). 